\title{
Bifurcation for Second-Order Hamiltonian Systems with Periodic Boundary Conditions
}

\author{
Francesca Faraci and Antonio Iannizzotto \\ Dipartimento di Matematica e Informatica, Università di Catania, Viale A. Doria 6, 95125 Catania, Italy \\ Correspondence should be addressed to Antonio Iannizzotto, iannizzotto@dmi.unict.it
}

Received 12 March 2007; Revised 21 June 2007; Accepted 18 December 2007

Recommended by Jean Mawhin

Through variational methods, we study nonautonomous systems of second-order ordinary differential equations with periodic boundary conditions. First, we deal with a nonlinear system, depending on a function $u$, and prove that the set of bifurcation points for the solutions of the system is not $\sigma$-compact. Then, we deal with a linear system depending on a real parameter $\lambda>0$ and on a function $u$, and prove that there exists $\lambda^{*}$ such that the set of the functions $u$, such that the system admits nontrivial solutions, contains an accumulation point.

Copyright (C) 2008 F. Faraci and A. Iannizzotto. This is an open access article distributed under the Creative Commons Attribution License, which permits unrestricted use, distribution, and reproduction in any medium, provided the original work is properly cited.

\section{Introduction}

In the present paper, we will deal with nonautonomous Hamiltonian systems with periodic boundary conditions, depending on a function $u$, of the following type:

$$
\begin{gathered}
\ddot{v}=A(t) v+\nabla F(t, v+u(t)) \quad \text { a.e. in } I, \\
v(0)-v(T)=\dot{v}(0)-\dot{v}(T)=0,
\end{gathered}
$$

where $I=[0, T]$ is a real interval, $A(\cdot)$ is an $N \times N$ symmetric, positive definite matrix; and the function $F: I \times \mathbb{R}^{N} \rightarrow \mathbb{R}$, referred to as the potential, is measurable with respect to the scalar variable and continuously differentiable with respect to the vector variable (note that by $\nabla F$ we will always mean the gradient of $F$ with respect to the vector variable).

The function $u$ is regarded as a parameter, and we are interested in studying the structure of the set of the solutions of $\left(\mathrm{N}_{u}\right)$, as $u$ varies in a suitable function space $X$. In particular, we will focus on those functions $u$ which are bifurcation points for the problem (see Definition 3.4 below), according to the very general definition given by Chow and Hale [1]. Actually, our 
result ensures that, under convenient assumptions on the potential $F$, the set of such bifurcation points is "large," that is, it is not $\sigma$-compact. Moreover, whenever $u$ is not a bifurcation point, $\left(\mathrm{N}_{u}\right)$ admits a finite number of solutions.

We will study $\left(\mathrm{N}_{u}\right)$ in the equivalent form of an equation in $X$, involving a nonlinear operator $\Phi$ from $X$ to itself: as the bifurcation points are exactly the singular values of $\Phi$, we will be able to apply a result established by Ricceri [2], assuring that the set of the singular values of $\Phi$ is not $\sigma$-compact. The equivalence between bifurcation points and singular values was already employed by Ďurikovič and Ďurikovičová [3], where a single second-order nonlinear differential equation is studied.

Our fundamental assumptions are that, for all $t \in I$, the function $F(t, \cdot)$ is positively homogeneous of degree $\alpha \in$ ]1,2[ and not quasiconvex (see condition (F3) below for a more precise statement). Homogeneity assumptions have already been used in the study of Hamiltonian system, for instance, Ben-Naoum et al. [4] proved the existence of a nonconstant solution, provided that $F$ satisfies certain sign assumptions and $F(t, \cdot)$ is positively homogeneous with degree $\alpha>1, \alpha \neq 2$. Our assumption that $\alpha \in] 1,2[$ places $F$ in the class of subquadratic potentials: in a similar framework, Tang and Wu proved in [5] the existence of a solution for a Hamiltonian system involving a matrix $A(\cdot)$ which is not necessarily positive definite.

We will also present a result (based on another theorem from [2]) in the framework of eigenvalue problems for linear second-order systems with periodic boundary conditions, depending on the function $u \in X$ and on the real parameter $\lambda>0$ of the following type:

$$
\begin{gathered}
\ddot{v}=A(t) v+\lambda H_{F}(t, u(t)) v \quad \text { a.e. in } I, \\
v(0)-v(T)=\dot{v}(0)-\dot{v}(T)=0,
\end{gathered}
$$

where $H_{F}(t, \cdot)$ denotes the Hessian matrix of some potential $F(t, \cdot)$, which is assumed to be twice differentiable. In this case, we replace the homogeneity condition by assuming a subquadratic growth of the potential, and obtain the existence of a real $\lambda^{*}$ such that the set of the functions $u$, such that the system $\left(L_{u, \lambda^{*}}\right)$ admits nontrivial solutions, contains an accumulation point.

\section{Preliminaries}

In this section, we introduce the common hypotheses and notation of the nonlinear and the linear cases, and recall some results which will be useful in the sequel.

Let $N \in \mathbb{N}(N>1), T>0, I=[0, T]$; let $A(\cdot)$ be an $N \times N$ real symmetric matrix, whose entries are functions $a_{i j} \in L^{\infty}(I)$, and assume that there exists $v>0$ such that, for a.e. $t \in I$ and every $x \in \mathbb{R}^{N}$,

$$
(A(t) x) \cdot x \geq v|x|^{2} .
$$

Following the monograph [6] by Mawhin and Willem, we denote by $H_{T}^{1}$ the space of the functions $u \in L^{2}\left(I, \mathbb{R}^{N}\right)$ with weak derivative $\dot{u} \in L^{2}\left(I, \mathbb{R}^{N}\right)$ and satisfying $u(T)=u(0)$. In particular, every $u \in H_{T}^{1}$ is an absolutely continuous function, hence it admits a classical derivative, equal to $\dot{u}$, a.e. in $I$. Due to the above assumptions on the matrix $A, H_{T}^{1}$ is endowed with a scalar product defined by putting for every $u, v \in H_{T}^{1}$,

$$
\langle u, v\rangle=\int_{0}^{T}[\dot{u}(t) \cdot \dot{v}(t)+(A(t) u(t)) \cdot v(t)] d t ;
$$


the induced norm is defined for every $u \in H_{T}^{1}$ by

$$
\|u\|=\sqrt{\int_{0}^{T}\left[|\dot{u}(t)|^{2}+(A(t) u(t)) \cdot u(t)\right] d t .}
$$

It is well known that $X:=\left(H_{T}^{1},\|\cdot\|\right)$ is an infinite-dimensional real Hilbert space, compactly embedded in $\mathcal{C}^{0}\left(I, \mathbb{R}^{N}\right)$; in particular, there exists $c>0$ such that

(C) $\|u\|_{\infty} \leq c\|u\|$ for every $u \in X$.

In the proofs of our results, we will employ some basic facts about nonlinear operators in Hilbert spaces, which we recall for the reader's convenience.

Let $X, Y$ be Banach spaces, $\Psi: X \rightarrow Y$ an operator. We recall that $\Psi$ is proper if, for any compact subset $C$ of $Y$, the set $\Psi^{-1}(C)$ is compact. We also recall the following result by Sadyrkhanov.

Theorem 2.1 (see [7, Theorem 1.1]). Let $X$ be an infinite-dimensional Hilbert space, $\Psi: X \rightarrow X a$ continuous, closed, nonconstant operator. Then, $\Psi$ is proper.

We also present the following statement of the domain invariance theorem.

Theorem 2.2 (see [8, Theorem 16.C]). Let $X$ be a Banach space, let $G$ be an open subset of $X$, let $\Psi_{0}: G \rightarrow X$ be a continuous, compact operator, and let $\Psi: G \rightarrow X$ be defined by $\Psi(u)=u+\Psi_{0}(u)$ for every $u \in G$. Assume that $\Psi$ is injective. Then, $\Psi$ is an open mapping.

We recall that $\Psi: X \rightarrow Y$ is Gâteaux differentiable in $X$ if there exists a mapping $\Psi^{\prime}$ : $X \rightarrow \mathcal{L}(X, Y)$ (by $\mathcal{L}(X, Y)$ we mean the space of bounded linear operators mapping $X$ into $Y$ ) such that, for all $u, v \in X$,

$$
\lim _{\tau \rightarrow 0^{+}} \frac{\Psi(u+\tau v)-\Psi(u)}{\tau}=\Psi^{\prime}(u)(v) ;
$$

if $\Psi^{\prime}$ is continuous, we will write $\Psi \in \mathcal{C}^{1}(X, Y)$.

We denote by $S_{\Psi}$ the set of the singular points of $\Psi$, that is, the set of all the points $u_{0}$ such that $\Psi$ is not a local homeomorphism at $u_{0}$. A point $v_{0} \in Y$ is said to be a singular value of $\Psi$ if it is the correspondent of some singular point, that is, if $v_{0} \in \Psi\left(S_{\Psi}\right)$. If, in addition, $\Psi \in \mathcal{C}^{1}(X, Y)$, we denote by $\widehat{S}_{\Psi}$ the set of all the points $u_{0} \in X$ such that $\Psi^{\prime}\left(u_{0}\right) \in \mathcal{L}(X, Y)$ is not surjective. Finally, we recall that a set is called $\sigma$-compact if it is the union of an at most countable family of compact sets.

Let $X$ be a Hilbert space, with scalar product $\langle\cdot, \cdot\rangle$ : we denote by $\left(X^{*},\|\cdot\|_{*}\right)$ the topological dual of $X$ and recall that, by the Riesz theorem, there exists a surjective linear isometry $\varphi \in$ $\mathcal{L}\left(X, X^{*}\right)$, satisfying the following equality for all $u, v \in X$ :

$$
\varphi(u)(v)=\langle u, v\rangle .
$$

Let $J: X \rightarrow \mathbb{R}$ be a Gâteaux differentiable functional, then its derivative is defined as a mapping $J^{\prime}: X \rightarrow X^{*}$, and we define an operator $\Phi: X \rightarrow X$ by putting for all $u \in X$,

$$
\Phi(u)=u+\varphi^{-1}\left(J^{\prime}(u)\right)
$$

note that, if $J \in \mathcal{C}^{1}(X, \mathbb{R})$, the mapping $\Phi$ is continuous.

The following result, due to Ricceri, will play a fundamental role in the study of problem $\left(\mathrm{N}_{u}\right)$. 
Theorem 2.3 (see [2, Theorem 1]). Let $X$ be an infinite-dimensional Hilbert space, $J \in \mathcal{C}^{1}(X, \mathbb{R})$. Assume that $J$ is sequentially weakly lower semicontinuous, not quasiconvex, and positively homogeneous of degree $\alpha \in] 1,2\left[\right.$; moreover, suppose that $\Phi$ is a closed mapping. Then, both sets $S_{\Phi}$ and $\Phi\left(S_{\Phi}\right)$ are not $\sigma$-compact.

Now, let $J: X \rightarrow \mathbb{R}$ be a twice-differentiable functional, then its second derivative (i.e., the derivative of $\left.J^{\prime}\right)$ is defined as a mapping $J^{\prime \prime}: X \rightarrow \mathcal{L}\left(X, X^{*}\right)$; for all $\lambda>0$, we define $\Phi_{\curlywedge}: X \rightarrow X$ by putting for all $u \in X$,

$$
\Phi_{\lambda}(u)=u+\lambda \varphi^{-1}\left(J^{\prime}(u)\right)
$$

We observe that $\Phi_{\lambda}$ is a Gâteaux differentiable mapping, whose derivative is a mapping $\Phi_{\lambda}^{\prime}$ : $X \rightarrow \mathcal{L}(X, X)$ expressed for all $u \in X$ by

$$
\Phi_{\lambda}^{\prime}(u)=\operatorname{Id}_{X}+\lambda \varphi^{-1} \circ\left(J^{\prime \prime}(u)\right)
$$

(this follows from the composite map formula, see Ambrosetti and Prodi [9, Chapter 1, Proposition 1.4]); in particular, if $J \in \mathcal{C}^{2}(X, \mathbb{R})$ (i.e., $J^{\prime} \in \mathcal{C}^{1}\left(X, X^{*}\right)$ ), then $\Phi_{\lambda} \in \mathcal{C}^{1}(X, X)$.

We will employ the following result, due to Ricceri as well, for the study of the problem $\left(\mathrm{L}_{u, \lambda}\right)$.

Theorem 2.4 (see [2, Theorem 3]). Let $X$ be an infinite-dimensional Hilbert space, let $J \in \mathcal{C}^{2}(X, \mathbb{R})$ be a non-quasiconvex functional. Assume that $J^{\prime}$ is compact and that

$$
\liminf _{\|u\| \rightarrow+\infty} \frac{J(u)}{\|u\|^{2}} \geq 0
$$

Moreover, suppose that

$$
\lim _{\|u\| \rightarrow+\infty}\left\|\Phi_{\curlywedge}(u)\right\| \longrightarrow+\infty
$$

Then, there exists $\lambda^{*}>0$ such that the set $\widehat{S}_{\Phi_{\lambda^{*}}}$ contains at least an accumulation point.

\section{The nonlinear case}

Let $F: I \times \mathbb{R}^{N} \rightarrow \mathbb{R}$ be a function satisfying the following conditions:

(F1) $F(\cdot, x)$ is measurable for every $x \in \mathbb{R}^{N}, F(t, \cdot) \in \mathcal{C}^{1}\left(\mathbb{R}^{N}, \mathbb{R}\right)$ for a.e. $t \in I$;

(F2) there exist $a \in C^{0}\left(\mathbb{R}^{+}, \mathbb{R}^{+}\right)$and a nonnegative $b \in L^{1}(I)$ such that for every $x \in \mathbb{R}^{N}$ and a.e. $t \in I$,

$$
\max \left\{|F(t, x)|,\left|\frac{\partial F}{\partial x_{i}}(t, x)\right|: i=1, \ldots, N\right\} \leq a(|x|) b(t)
$$

(F3) there exist $\left.\rho, \sigma \in \mathbb{R}, x_{1}, x_{2} \in \mathbb{R}^{N}, \tau \in\right] 0,1\left[\right.$, and a closed interval $\left.I_{0} \subset\right] 0, T$ [ such that for a.e. $t \in I_{0}$,

$$
\max \left\{F\left(t, x_{1}\right), F\left(t, x_{2}\right)\right\} \leq \rho<\sigma \leq F\left(t, \tau x_{1}+(1-\tau) x_{2}\right)
$$


(F4) there exists $\alpha \in] 1,2\left[\right.$ such that for every $\mu>0, x \in \mathbb{R}^{N}$, and a.e. $t \in I$,

$$
F(t, \mu x)=\mu^{\alpha} F(t, x)
$$

In (F2). we can assume $a$ increasing, without loss of generality. Note that from (F4) it follows that $F(t, 0)=0$ a.e. in $I$.

In this section, we deal with the nonlinear problem $\left(\mathrm{N}_{u}\right)$, depending on the function $u \in X$. We recall that, for every $u \in X$, a solution of $\left(\mathrm{N}_{u}\right)$ is a function $v \in X$ such that for every $w \in X$

$$
\int_{0}^{T}[\dot{v}(t) \cdot \dot{w}(t)+(A(t) v(t)) \cdot w(t)+\nabla F(t, v(t)+u(t)) \cdot w(t)] d t=0
$$

We observe that, by the results of [6, (Section 1.4)], whenever $v \in X$ is a solution of $\left(\mathrm{N}_{u}\right)$ in the above sense, actually $v \in \mathcal{C}^{1}\left(I, \mathbb{R}^{\mathbb{N}}\right)$ with derivative $\dot{v} \in X$. Thus, clearly $\dot{v}(T)=\dot{v}(0)$; moreover, $\dot{v}$ is absolutely continuous, hence the second derivative $\ddot{v}$ exists a.e. in $I$ and satisfies

$$
\ddot{v}(t)=A(t) v(t)+\nabla F(t, v(t)+u(t)) \quad \text { for a.e. } t \in I .
$$

Now, we are going to introduce a suitable variational setting for the problem $\left(\mathrm{N}_{u}\right)$. Firstly, we put, for every $u \in X$,

$$
J(u)=\int_{0}^{T} F(t, u(t)) d t
$$

The following lemma describes the properties of the functional $J$.

Lemma 3.1. Let (F1), (F2), (F3), and (F4) be satisfied. Then, the functional $J \in \mathcal{C}^{1}(\mathrm{X}, \mathbb{R})$ with compact derivative $J^{\prime}: X \rightarrow X^{*}$. Moreover, $J$ is not quasiconvex.

Proof. By standard arguments, it is proved that $J \in \mathcal{C}^{1}(X, \mathbb{R})$, its derivative is an operator $J^{\prime}$ : $X \rightarrow X^{*}$, expressed by

$$
J^{\prime}(u)(v)=\int_{0}^{T} \nabla F(t, u(t)) \cdot v(t) d t
$$

for every $u, v \in X$. We are now going to prove that the map $J^{\prime}$ is compact: let $\left(u_{k}\right)$ be a bounded sequence in $X$, then there exist a subsequence, still denoted by $\left(u_{k}\right)$, and some $u \in X$ such that $\left\|u_{k}-u\right\|_{\infty} \rightarrow 0$; hence for all $k \in \mathbb{N}$,

$$
\left\|J^{\prime}\left(u_{k}\right)-J^{\prime}(u)\right\|_{*} \leq c \int_{0}^{T}\left|\nabla F\left(t, u_{k}(t)\right)-\nabla F(t, u(t))\right| d t,
$$

where $c$ is as in (C). Conditions (F1) and (F2) ensure that the right-hand side tends to zero as $k \rightarrow \infty$, by an application of the Lebesgue theorem.

Let us prove now that the functional $J$ is not quasiconvex, that is, it has a nonconvex sublevel set. Define $M=\max \left\{\left|x_{1}\right|,\left|x_{2}\right|\right\}$. Since $b \in L^{1}(I)$, there exists $\delta>0$ such that for every 
measurable set $\Omega \subseteq I$ with $m(\Omega)<\delta$ (where $m(\Omega)$ denotes the Lebesgue measure of $\Omega$ ), we have

$$
\int_{\Omega} b(t) d t<\frac{(\sigma-\rho) m\left(I_{0}\right)}{2 a(M)}
$$

Without loss of generality, we can choose $\delta$ such that

$$
\left.I_{1}:=\left[\inf \left(I_{0}\right)-\frac{\delta}{3}, \sup \left(I_{0}\right)+\frac{\delta}{3}\right] \subset\right] 0, T[,
$$

so that $m\left(I_{1} \backslash I_{0}\right)<\delta$, and functions $u_{i} \in C^{\infty}(] 0, T[)$ for $i=1,2$, such that $u_{i}(t)=x_{i}$ for every $t \in I_{0}$ and $u_{i}(t)=0$ for every $t \in I \backslash I_{1}$, satisfying the condition $\left\|u_{i}\right\|_{\infty}=\left|x_{i}\right|$. Since $F(t, 0)=0$ due to (F4), for $i=1,2$, we have

$$
J\left(u_{i}\right)=\int_{I_{0}} F\left(t, x_{i}\right) d t+\int_{I_{1} \backslash I_{0}} F\left(t, u_{i}(t)\right) d t \leq \rho m\left(I_{0}\right)+a(M) \int_{I_{1} \backslash I_{0}} b(t) d t<\frac{(\sigma+\rho) m\left(I_{0}\right)}{2} .
$$

Moreover, if $\bar{u}=\tau u_{1}+(1-\tau) u_{2}$, then an analogous argument leads to

$$
J(\bar{u})>\frac{(\sigma+\rho) m\left(I_{0}\right)}{2} .
$$

Thus, it is proved that the set $J^{-1}(]-\infty,(\sigma+\rho) m\left(I_{0}\right) / 2[)$ is not convex.

Lemma 3.2. Let (F1), (F2), and (F4) be satisfied. Then, $J$ and $J$ are positively homogeneous with exponents $\alpha, \alpha-1$, respectively (in particular, they both vanish at 0). Moreover,

$$
\lim _{\|u\| \rightarrow+\infty} \frac{\left\|J^{\prime}(u)\right\|_{*}}{\|u\|}=0
$$

Proof. The homogeneity properties of $J$ and $J^{\prime}$ are easily obtained from (F4). Condition (3.13) is quickly deduced as well, as we recall that $\alpha-1<1$.

We define the operator $\Phi: X \rightarrow X$ by putting, for every $u \in X$,

$$
\Phi(u)=u+\varphi^{-1}\left(J^{\prime}(u)\right)
$$

(where $\varphi$ is as in Section 2). The next lemma yields some properties of the operator $\Phi$.

Lemma 3.3. Let (F1), (F2), and (F4) be satisfied. Then, $\Phi$ satisfies the condition

$$
\lim _{\|u\| \rightarrow+\infty}\|\Phi(u)\|=+\infty,
$$

and it is closed and proper. 
Proof. Since $\varphi$ is an isometry, for all $u \in X$, we get

$$
\|\Phi(u)\|=\left\|u+\varphi^{-1}\left(J^{\prime}(u)\right)\right\| \geq\|u\|\left(1-\frac{\left\|J^{\prime}(u)\right\|_{*}}{\|u\|}\right),
$$

so from condition (3.13) we deduce (3.15).

Now we prove that $\Phi$ is a closed mapping. Let $C$ be a closed subset of $X$, we need to prove that $\Phi(C)$ is closed as well. Assume that $\left\{u_{n}\right\}$ is a sequence in $C$ such that $\left\{\Phi\left(u_{n}\right)\right\}$ converges to some $v \in X$. Then, by (3.15), $\left\{u_{n}\right\}$ is bounded; since $J^{\prime}$ is compact (Lemma 3.1) and $\varphi$ is surjective, there exists a subsequence $\left\{u_{n_{k}}\right\}$ such that $\left\{J^{\prime}\left(u_{n_{k}}\right)\right\}$ converges to $\varphi(w)$ for some $w \in X$. We get

$$
u_{n_{k}}=\Phi\left(u_{n_{k}}\right)-\varphi^{-1}\left(J^{\prime}\left(u_{n_{k}}\right)\right) \longrightarrow v-w,
$$

hence, $v-w \in C$. Since $J^{\prime}$ is continuous (Lemma 3.1), $J^{\prime}(v-w)=\varphi(w)$, so

$$
\Phi(v-w)=v-w+\varphi^{-1}\left(J^{\prime}(v-w)\right)=v
$$

which implies $v \in \Phi(C)$.

Finally, we note that $\Phi$ is continuous and is not constant, then by Theorem 2.1, $\Phi$ is proper.

The operator $\Phi$ provides the desired variational setting for $\left(\mathrm{N}_{u}\right)$. Indeed, let us define the set

$$
\Sigma=\left\{(v, u) \in X \times X: v \text { is a solution of }\left(\mathrm{N}_{u}\right)\right\}
$$

and for every $u \in X$,

$$
\Sigma_{u}=\{v \in X:(v, u) \in \Sigma\}
$$

By the definition of $\Phi$, it is clear that, for every $u \in X$,

$$
\Sigma_{u}=\{v \in X: \Phi(v+u)=u\} .
$$

Indeed, for all $v \in X$, we have

$$
\Phi(v+u)=u
$$

if and only if for all $w \in X$,

$$
0=\left\langle v+\varphi^{-1}\left(J^{\prime}(v+u)\right), w\right\rangle=\langle v, w\rangle+J^{\prime}(v+u)(w),
$$

which is equivalent to $v \in \Sigma_{u}$.

Next, we give the definition of bifurcation point for $\Sigma$, equivalent to the one of [1, page 2]. 
Definition 3.4. A bifurcation point for $\Sigma$ is a function $u \in X$ such that there exist $v \in \Sigma_{u}$ and sequences $\left(u_{n}\right),\left(v_{n}^{1}\right)$, and $\left(v_{n}^{2}\right)$ in $X$ such that $v_{n}^{1}, v_{n}^{2} \in \Sigma_{u_{n}}, v_{n}^{1} \neq v_{n}^{2}$ for every $n \in \mathbb{N}$, and

$$
\lim _{n} u_{n}=u, \quad \lim _{n} v_{n}^{1}=\lim _{n} v_{n}^{2}=v .
$$

As pointed out in the introduction, we are interested in the "size" of the set of the bifurcation points for $\Sigma$. Our main result, which is based on Theorem 2.3, ensures that such set is not $\sigma$ compact and that whenever $u$ is not a bifurcation point, the set $\Sigma_{u}$ is nonempty and finite. The precise statement is the following.

Theorem 3.5. Let (F1), (F2), (F3), and (F4) be satisfied. Then, the following assertions hold:

(I) the set of the bifurcation points for $\Sigma$ is closed and not $\sigma$-compact;

(II) for every $u \in X$ which is not a bifurcation point for $\Sigma$, the set $\Sigma_{u}$ is nonempty and finite.

Proof. In order to prove (I), we are going to apply Theorem 2.3, all of its hypotheses are fulfilled due to Lemmas 3.1, 3.2, and 3.3 (in particular, we point out that, since $J^{\prime}$ is compact, $J$ turns out to be sequentially weakly continuous). Thus, the sets $S_{\Phi}$ and $\Phi\left(S_{\Phi}\right)$ are not $\sigma$-compact. Moreover, from the definition of a singular point, it is immediately deduced that $S_{\Phi}$ is closed; since $\Phi$ is a closed operator, $\Phi\left(S_{\Phi}\right)$ is closed too.

All that remains to prove is that $\Phi\left(S_{\Phi}\right)$ is the set of the bifurcation points for $\Sigma$.

Indeed, choose $u \in \Phi\left(S_{\Phi}\right)$, then by (3.21), there is a $v \in \Sigma_{u}$ such that $v+u \in S_{\Phi}$. Moreover, for every $n \in \mathbb{N}$, denote $B_{n}$ the open ball in $X$ centered in $v+u$ with radius $1 / n$ : since $v+u \in S_{\Phi}$, $\Phi: B_{n} \rightarrow \Phi\left(B_{n}\right)$ is not a homeomorphism.

Now we prove that $\Phi: B_{n} \rightarrow \Phi\left(B_{n}\right)$ is not injective, arguing by contradiction. Assume that $\Phi: B_{n} \rightarrow \Phi\left(B_{n}\right)$ is injective, we already know that $J^{\prime}: X \rightarrow X^{*}$ is a compact operator, so clearly $\varphi^{-1} \circ J^{\prime}: X \rightarrow X$ is compact as well; thus, by Theorem 2.2, $\Phi: B_{n} \rightarrow \Phi\left(B_{n}\right)$ would be open, hence a homeomorphism, which is a contradiction.

Thus, there are $w_{n}^{1}, w_{n}^{2} \in B_{n}$ with $w_{n}^{1} \neq w_{n}^{2}$ such that $\Phi\left(w_{n}^{1}\right)=\Phi\left(w_{n}^{2}\right)=: u_{n}$. Clearly,

$$
\lim _{n} w_{n}^{1}=\lim _{n} w_{n}^{2}=v+u, \quad \lim _{n} u_{n}=u
$$

so, denoting $v_{n}^{i}=w_{n}^{i}-u_{n}$ for $i=1,2$, Definition 3.4 is fulfilled and $u$ is a bifurcation point for $\Sigma$.

On the other hand, choose $u \in X \backslash \Phi\left(S_{\Phi}\right)$, then by (3.21), it clearly follows that

$$
\Sigma_{u} \cap\left(S_{\Phi}-u\right)=\varnothing
$$

Then, $u$ is not a bifurcation point for $\Sigma$; indeed, for every $v \in \Sigma_{u}$ we have $v+u \notin S_{\Phi}$, that is, $\Phi$ is a local homeomorphism in $v+u$; in particular, there exists an open neighborhood $V$ of $v$, such that the restriction of $\Phi$ to $V+u$ is injective, hence $u$ cannot comply with Definition 3.4.

Now we prove (II). Choose again $u \in X \backslash \Phi\left(S_{\Phi}\right)$. Let us define an energy functional $E$ by putting for every $v \in X$,

$$
E(v)=\frac{\|v\|^{2}}{2}+J(v+u)
$$


it is easily seen that $E \in \mathcal{C}^{1}(X, \mathbb{R})$ and its derivative satisfies, for all $v, w \in X$, the following equality:

$$
E^{\prime}(v)(w)=\langle v, w\rangle+J^{\prime}(v+u)(w)=\langle\Phi(v+u)-u, w\rangle
$$

so, by (3.21), $\Sigma_{u}$ is the set of the critical points of $E$.

We prove now that $E$ is coercive; with this aim in mind, we note that $J$, as a $\mathcal{C}^{1}$ functional with compact derivative, is sequentially weakly continuous, hence its restriction to the closed unit ball admits minimum $k \in \mathbb{R}$; for $\|v\|$ big enough, from (F4), we easily get

$$
E(v)=\frac{\|v\|^{2}}{2}+\|v+u\|^{\alpha} J\left(\frac{v+u}{\|v+u\|}\right) \geq \frac{\|v\|^{2}}{2}+k\|v+u\|^{\alpha},
$$

and the latter goes to $+\infty$ as $\|v\| \rightarrow+\infty$ (since $\alpha<2$ ).

We observe, also, that $E$ is sequentially weakly l.s.c. Thus, $E$ admits a global minimum, that is, $\Sigma_{u} \neq \varnothing$.

Finally, we prove that $\Sigma_{u}$ has a finite number of elements: first, recalling that $\Phi$ is proper (Lemma 3.3), we observe that $\Sigma_{u}$ is compact due to (3.21). Besides, $\Sigma_{u}$ is a discrete set. Indeed, for every $v \in \Sigma_{u}$, we have already observed that $v$ admits an open neighborhood $V$ such that the restriction of $\Phi$ to $V+u$ is injective, in particular

$$
V \cap \Sigma_{u}=\{v\}
$$

Being compact and discrete, $\Sigma_{u}$ is finite, which concludes the proof.

Before concluding this section, we give an example of application of Theorem 3.5 to a system of two equations.

Example 3.6. Let $N=2, A(\cdot)=\left[a_{i j}(\cdot)\right]$ be a $2 \times 2$ matrix as in Section 2, let $\left.\alpha \in\right] 1,2[$ be a real number, and consider the following problem, depending on the function $u \in X$ :

$$
\begin{aligned}
& \ddot{v}_{1}=a_{11}(t) v_{1}+a_{12}(t) v_{2}+\alpha\left|v_{1}+u_{1}(t)\right|^{\alpha-2}\left(v_{1}+u_{1}(t)\right) \text { a.e. in } I, \\
& \ddot{v}_{2}=a_{21}(t) v_{1}+a_{22}(t) v_{2}-\alpha\left|v_{2}+u_{2}(t)\right|^{\alpha-2}\left(v_{2}+u_{2}(t)\right) \text { a.e. in } I \text {, } \\
& v_{1}(0)-v_{1}(T)=\dot{v}_{1}(0)-\dot{v}_{1}(T)=0, \\
& v_{2}(0)-v_{2}(T)=\dot{v}_{2}(0)-\dot{v}_{2}(T)=0 \text {. }
\end{aligned}
$$

We are led to the study of the potential $F: \mathbb{R}^{2} \rightarrow \mathbb{R}$ defined by

$$
F\left(x_{1}, x_{2}\right)=\left|x_{1}\right|^{\alpha}-\left|x_{2}\right|^{\alpha},
$$

which satisfies all the assumptions of Theorem 3.5. Thus, the set of bifurcation points related to the system is not $\sigma$-compact, and for every $u \in X$ which is not a bifurcation point, the set of solutions of the problem is nonempty and finite. 


\section{The linear case}

Let $F: I \times \mathbb{R}^{N} \rightarrow \mathbb{R}$ be a function satisfying the following conditions:

(F5) $F(\cdot, x)$ is measurable for every $x \in \mathbb{R}^{N}, F(t, \cdot) \in \mathcal{C}^{2}\left(\mathbb{R}^{N}, \mathbb{R}\right)$, and $F(t, 0)=0$ for a.e. $t \in I$;

(F6) there exist $a \in \mathcal{C}^{0}\left(\mathbb{R}^{+}, \mathbb{R}^{+}\right)$and a nonnegative $b \in L^{1}(I)$ such that for every $x \in \mathbb{R}^{N}$ and a.e. $t \in I$,

$$
\max \left\{|F(t, x)|,\left|\frac{\partial F}{\partial x_{i}}(t, x)\right|,\left|\frac{\partial^{2} F}{\partial x_{i} \partial x_{j}}(t, x)\right|: i, j=1, \ldots, N\right\} \leq a(|x|) b(t) ;
$$

(F7) $\lim _{|x| \rightarrow+\infty} \operatorname{ess}_{\sup }(|\nabla F(t, x)| /|x|)=0$.

As above, in (F6) we can assume $a$ increasing. Besides, in this section we will also assume that condition (F3), stated as in Section 3, is fulfilled. In the sequel, for every $(t, x) \in I \times \mathbb{R}^{N}$, we denote by $H_{F}(t, x)$ the Hessian matrix of $F(t, \cdot)$ in $x$.

In this section, we deal with the linear problem $\left(\mathrm{L}_{u, \lambda}\right)$, depending on the function $u \in X$ and on the real parameter $\lambda>0$. We recall that, for every $u \in X$ and $\lambda>0$, a solution of $\left(L_{u, \lambda}\right)$ is a function $v \in X$ such that for every $w \in X$,

$$
\int_{0}^{T}\left[\dot{v}(t) \cdot \dot{w}(t)+(A(t) v(t)) \cdot w(t)+\lambda\left(H_{F}(t, u(t)) v(t)\right) \cdot w(t)\right] d t=0
$$

(the meaning of such definition being the same as in Section 3). For every $\lambda>0$, we denote by $R_{\lambda}$ the set of all $u \in X$ such that $\left(\mathrm{L}_{u, \lambda}\right)$ admits at least a nonzero solution.

We define the functional $J$ over $X$ as in Section 3 , and collect its properties in the following lemma.

Lemma 4.1. Let (F3), (F5), (F6), and (F7) be satisfied. Then, $J \in \mathcal{C}^{2}(X, \mathbb{R})$, its first derivative $J^{\prime}$ : $X \rightarrow X^{*}$ is a compact mapping and its second derivative $J^{\prime \prime}: X \rightarrow \mathcal{L}\left(X, X^{*}\right)$ is such that $J^{\prime \prime}(u)$ is a compact linear operator for all $u \in X$. Moreover, $J$ is not quasiconvex and satisfies (3.13) and

$$
\lim _{\|u\| \rightarrow+\infty} \frac{J(u)}{\|u\|^{2}}=0 .
$$

Proof. Clearly, (F5) and (F6) imply (F1) and (F2), respectively, so as in Lemma 3.1, $J \in \mathcal{C}^{1}(\mathrm{X}, \mathbb{R})$ with a compact derivative $J^{\prime}: X \rightarrow X^{*}$ (note that, in Lemma 3.1, the homogeneity assumption (F4) was employed only to deduce $F(\cdot, 0)=0$, which here is explicitly assumed in (F5)). Also, from (F5) and (F6), it is easily deduced that the operator $J^{\prime}$ is continuously differentiable and its derivative is a mapping $J^{\prime \prime}: X \rightarrow \mathcal{L}\left(X, X^{*}\right)$ expressed by

$$
J^{\prime \prime}(u)(v)(w)=\int_{0}^{T}\left(H_{F}(t, u(t)) v(t)\right) \cdot w(t) d t
$$

for all $u, v, w \in X$.

Next we prove that, for all $u \in X, J^{\prime \prime}(u)$ is a compact linear operator. Let $\left\{v_{n}\right\}$ be a sequence in $X$ with $\left\|v_{n}\right\| \leq M$ for all $n \in \mathbb{N}(M>0)$; we wish to prove that $\left\{J^{\prime \prime}(u)\left(v_{n}\right)\right\}$ admits 
a subsequence which converges to some element of $X^{*}$. To this end, we fix $\varepsilon>0$ and observe that there exists $\delta>0$ such that

$$
\frac{\left\|J^{\prime}(u+v)-J^{\prime}(u)-J^{\prime \prime}(u)(v)\right\|_{*}}{\|v\|}<\frac{\varepsilon}{3 M}
$$

for all $v \in X$ with $\|v\|<\delta$. We choose now $\mu \in] 0, \delta / M\left[\right.$ and consider the sequence $\left\{u+\mu v_{n}\right\}$, which is bounded; by the compactness of $J^{\prime}$, we can assume that (up to a subsequence) $\left\{J^{\prime}(u+\right.$ $\left.\mu v_{n}\right)$ \} converges in $X^{*}$, hence in particular, it is a Cauchy sequence. So there is $v \in \mathbb{N}$ such that

$$
\left\|J^{\prime}\left(u-\mu v_{n}\right)-J^{\prime}\left(u-\mu v_{m}\right)\right\|_{*}<\frac{\varepsilon \mu}{3}
$$

for all $n, m>v$. It is easily seen that

$$
\left\|J^{\prime \prime}(u)\left(v_{n}\right)-J^{\prime \prime}(u)\left(v_{m}\right)\right\|_{*}<\varepsilon
$$

for all $n, m>v$, so since $X^{*}$ is a complete metric space, $\left\{J^{\prime \prime}(u)\left(v_{n}\right)\right\}$ is convergent.

From (F7), through an application of the mean value theorem, we obtain

$$
\lim _{|x| \rightarrow+\infty} \operatorname{ess} \sup \frac{F(t, x)}{|x|^{2}}=0 .
$$

The asymptotic behaviors of $J$ and $J^{\prime}$ are easily deduced from those of $F, \nabla F$, so (3.13) and (4.3) hold.

Finally, the existence of a nonconvex sublevel set of $J$ is proved as in Lemma 3.1.

For every $\lambda>0$, we define the mapping $\Phi_{\lambda}: X \rightarrow X$ by putting for all $u \in X$,

$$
\Phi_{\lambda}(u)=u+\lambda \varphi^{-1}\left(J^{\prime}(u)\right)
$$

and show its properties in the next lemma.

Lemma 4.2. Let (F5), (F6), and (F7) be satisfied. Then, for every $\lambda>0, \Phi_{\lambda} \in \mathcal{C}^{1}(X, X)$ and the following condition holds:

$$
\lim _{\|u\| \rightarrow+\infty}\left\|\Phi_{\lambda}(u)\right\|=+\infty .
$$

Proof. Fix $\lambda>0$. Since $J \in \mathcal{C}^{2}(X, \mathbb{R})$, from what is observed in Section 2 , we deduce that $\Phi_{\lambda} \in$ $\mathcal{C}^{1}(X, X)$ and its derivative is a mapping $\Phi_{\lambda}^{\prime}: X \rightarrow \mathcal{L}(X, X)$ defined by

$$
\Phi_{\lambda}^{\prime}(u)=\operatorname{Id}_{X}+\lambda \varphi^{-1} \circ\left(J^{\prime \prime}(u)\right)
$$

for all $u \in X$. In order to achieve (4.10), we proceed as in the proof of Lemma 3.3, using (3.13).

The next theorem describes the structure of the set $R_{\lambda}$ for a convenient $\lambda>0$.

Theorem 4.3. Let (F3), (F5), (F6), and (F7) be satisfied. Then, there exists $\lambda^{*}>0$ such that $R_{\lambda^{*}}$ contains at least one accumulation point. 
Proof. The assumptions of Theorem 2.4 are satisfied due to Lemmas 4.1 and 4.2 , hence there exists $\lambda^{*}>0$ such that the set $\widehat{S}_{\Phi_{\lambda^{*}}}$, consisting of the points $u \in X$ such that $\Phi_{\lambda^{*}}^{\prime}(u)$ is not surjective, has an accumulation point $u^{*} \in X$.

We note that $\widehat{S}_{\Phi_{\lambda^{*}}}$ is a closed set. To prove this assertion, we observe that the set $\mathcal{A}$ of all surjective bounded linear operators from $X$ into itself is open in $\mathcal{L}(X, X)$ (see Dieudonné [10, Théorème 1]).

Besides, $\Phi_{\lambda^{*}}^{\prime}: X \rightarrow \mathcal{L}(X, X)$ is a continuous mapping, so the set

$$
X \backslash \widehat{S}_{\Phi_{\lambda^{*}}}=\left\{u \in X: \Phi_{\lambda^{*}}^{\prime}(u) \in \mathcal{A}\right\}
$$

is open. Thus, $u^{*} \in \widehat{S}_{\Phi_{\lambda^{*}}}$.

To conclude the proof, it remains to show that for all $\lambda>0$

$$
\widehat{S}_{\Phi_{\lambda}}=R_{\curlywedge} .
$$

With this aim in mind, we fix $u \in X$ and note that the linear operator

$$
\Phi_{\lambda}^{\prime}(u)=\operatorname{Id}_{X}+\lambda \varphi^{-1} \circ\left(J^{\prime \prime}(u)\right)
$$

satisfies the hypotheses of the Fredholm alternative theorem ([9, Theorem 0.1]). Indeed, by Lemma 4.1, $\varphi^{-1} \circ\left(J^{\prime \prime}(u)\right) \in \mathcal{L}(X, X)$ is compact (recall that $\varphi^{-1}$ is a linear isometry). Thus, $\Phi_{\lambda}^{\prime}(u) \in \mathcal{L}(X, X)$ is injective iff it is surjective. Hence, $u$ belongs to $\widehat{S}_{\Phi_{\lambda}}$ iff $\Phi_{\lambda}^{\prime}(u)$ is not injective, that is, iff there exists $v \in X \backslash\{0\}$ satisfying $\Phi_{\lambda}^{\prime}(u)(v)=0$. Resuming, $u$ lies in $\widehat{S}_{\Phi_{\curlywedge}}$ iff there exists $v \in X \backslash\{0\}$ such that for all $w \in X$

$$
\langle v, w\rangle+\lambda J^{\prime \prime}(u)(v)(w)=0,
$$

that is, $v$ is a solution of $\left(\mathrm{L}_{u, \lambda}\right)$. Thus, (4.13) is proved and we may conclude that the set $R_{\lambda^{*}}$ contains an accumulation point.

We conclude by presenting the following example.

Example 4.4. Let $N>1, A(\cdot)=\left[a_{i j}(\cdot)\right]$ be an $N \times N$ matrix as in Section 2, and consider the following problem, depending on the function $u \in X$ and on the real parameter $\lambda>0$ :

$$
\begin{gathered}
\ddot{v}_{i}=\sum_{j=1}^{N}\left[a_{i j}(t)+2 \lambda e^{-|u(t)|^{2}}\left(2 u_{i}(t) u_{j}(t)-\delta_{i j}\right)\right] v_{j} \text { a.e. in } I, \quad i=1, \ldots, N, \\
v_{i}(0)-v_{i}(T)=\dot{v}_{i}(0)-\dot{v}_{i}(T)=0, \quad i=1, \ldots, N
\end{gathered}
$$

(here $\delta_{i j}$ is the Kronecker symbol). We are led to the study of the potential $F: \mathbb{R}^{N} \rightarrow \mathbb{R}$ defined by

$$
F(x)=e^{-|x|^{2}}-1
$$

which satisfies all the assumptions of Theorem 4.3. Thus, there exists $\lambda^{*}>0$ such that the set $R_{\lambda^{*}}$ contains at least one accumulation point. 


\section{References}

[1] S. N. Chow and J. K. Hale, Methods of Bifurcation Theory, vol. 251 of Grundlehren der Mathematischen Wissenschaften, Springer, New York, NY, USA, 1982.

[2] B. Ricceri, "On the singular set of certain potential operators in Hilbert spaces," in Differential Equations, Chaos and Variational Problems, V. Staicu, Ed., Progress in Nonlinear Differential Equations and Applications, pp. 377-391, Birkhäuser, Boston, Mass, USA, 2007.

[3] V. Durikovič and M. Durikovičová, "On the solutions of nonlinear initial-boundary value problems," Abstract and Applied Analysis, vol. 2004, no. 5, pp. 407-424, 2004.

[4] A. K. Ben-Naoum, C. Troestler, and M. Willem, "Existence and multiplicity results for homogeneous second order differential equations," Journal of Differential Equations, vol. 112, no. 1, pp. 239-249, 1994.

[5] C.-L. Tang and X.-P. Wu, "Periodic solutions for a class of nonautonomous subquadratic second order Hamiltonian systems," Journal of Mathematical Analysis and Applications, vol. 275, no. 2, pp. 870-882, 2002.

[6] J. Mawhin and M. Willem, Critical Point Theory and Hamiltonian Systems, vol. 74 of Applied Mathematical Sciences, Springer, New York, NY, USA, 1989.

[7] R. S. Sadyrkhanov, "On infinite-dimensional features of proper and closed mappings," Proceedings of the American Mathematical Society, vol. 98, no. 4, pp. 643-648, 1986.

[8] E. Zeidler, Nonlinear Functional Analysis and Its Applications. I, Springer, New York, NY, USA, 1986.

[9] A. Ambrosetti and G. Prodi, A Primer of Nonlinear Analysis, vol. 34 of Cambridge Studies in Advanced Mathematics, Cambridge University Press, Cambridge, UK, 1995.

[10] J. Dieudonné, "Sur les homomorphismes d'espaces normés," Bulletin des Sciences Mathématiques, vol. 67, pp. 72-84, 1943. 


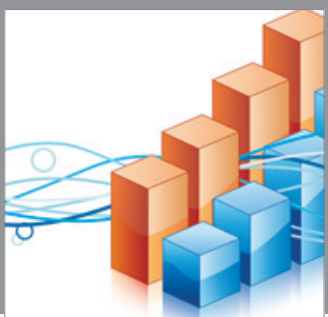

Advances in

Operations Research

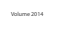

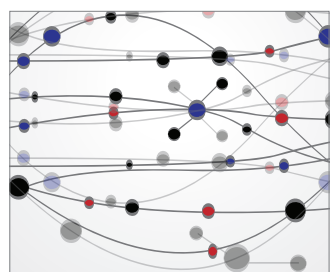

\section{The Scientific} World Journal
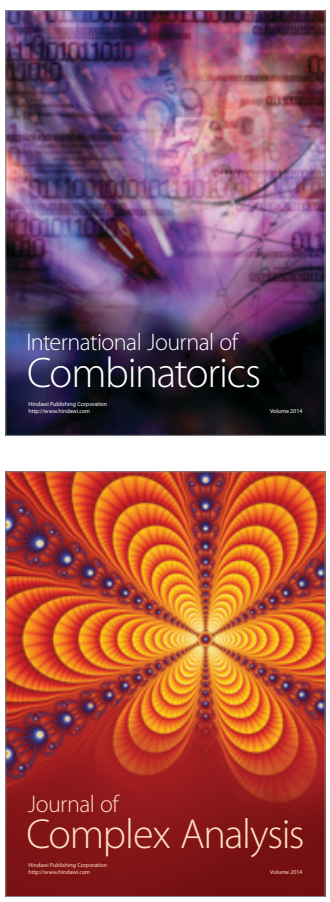

International Journal of

Mathematics and

Mathematical

Sciences
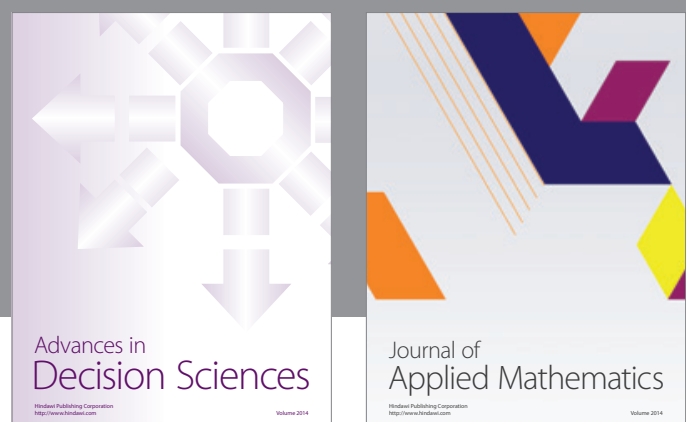

Journal of

Applied Mathematics
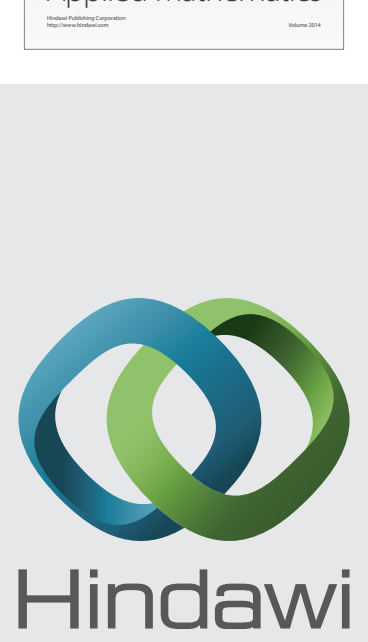

Submit your manuscripts at http://www.hindawi.com
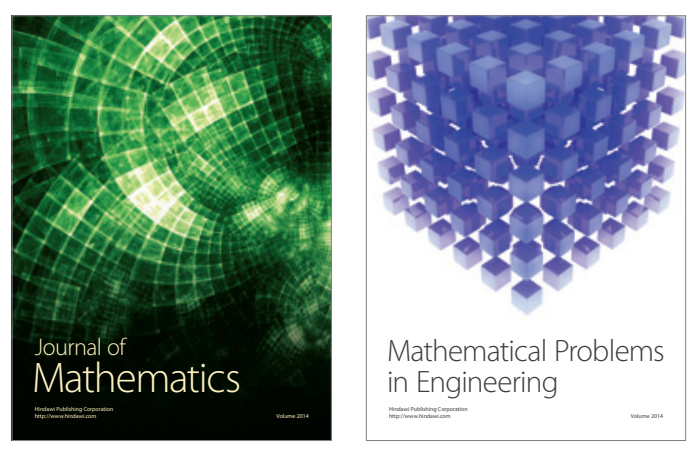

Mathematical Problems in Engineering
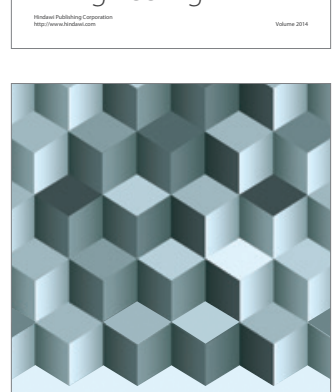

Journal of

Function Spaces
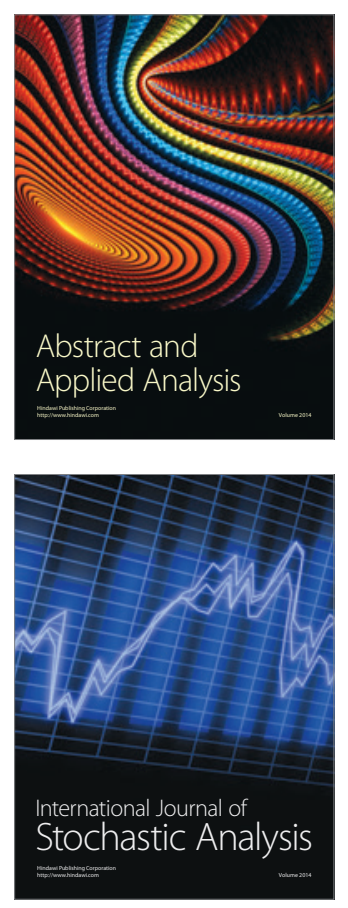

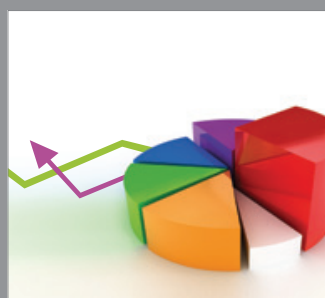

ournal of

Probability and Statistics

Promensencen
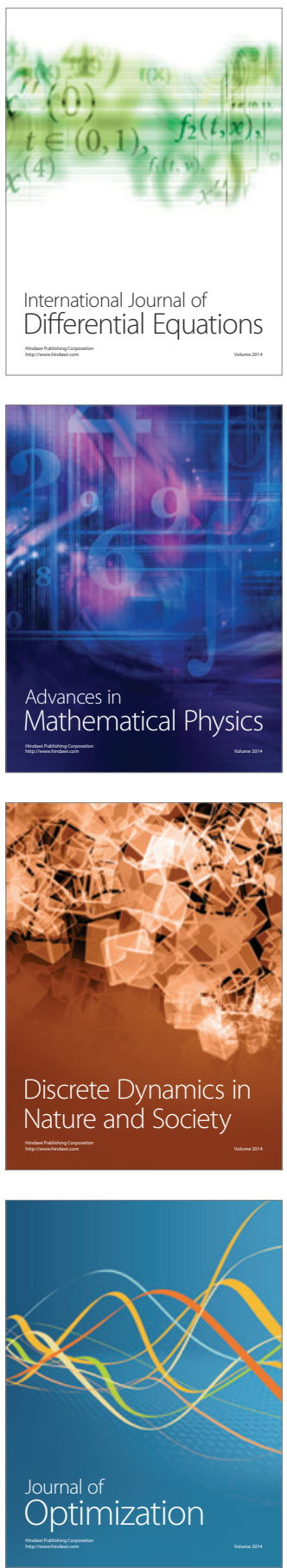